\title{
Long non-coding RNAs in infection biology
}

\author{
Vinod Scaria ${ }^{1 *}$ and Ayesha Pasha ${ }^{2}$ \\ GN Ramachandran Knowledge Center for Genome Informatics, Institute of Genomics and Integrative Biology, Council of Scientific and Industrial Research, Delhi, \\ India \\ ${ }^{2}$ Open Source Drug Discovery Unit, Council of Scientific and Industrial Research, New Delhi, India
}

Edited by:

Rami I. Aqeilan, Hebrew

University-Hadassah Medical School,

Israel

Reviewed by:

Noam Shomron, Tel Aviv University, Israel

Kuan Teh Jeang, National Institutes of Health, USA

\section{*Correspondence:}

Vinod Scaria, GN Ramachandran Knowledge Center for Genome Informatics, Institute of Genomics and Integrative Biology, Council of Scientific and Industrial Research, Mall Road, Delhi 110007, India. e-mail:vinods@igib.in
Long non-coding RNA have emerged as an increasingly well studied subset of non-coding RNAs (ncRNAs) following their recent discovery in a number of organisms including humans and characterization of their functional and regulatory roles in variety of distinct cellular mechanisms. The recent annotations of long ncRNAs in humans peg their numbers as similar to protein-coding genes. However, despite the rapid advancements in the field the functional characterization and biological roles of most of the long ncRNAs still remain unidentified, although some candidate long ncRNAs have been extensively studied for their roles in cancers and biological phenomena such as X-inactivation and epigenetic regulation of genes. A number of recent reports suggest an exciting possibility of long ncRNAs mediating host response and immune function, suggesting an elaborate network of regulatory interactions mediated through ncRNAs in infection. The present role of long ncRNAs in host-pathogen cross talk is limited to a handful of mechanistically distinct examples. The current commentary chronicles the findings of these reports on the role of long ncRNAs in infection biology and further highlights the bottlenecks and future directions toward understanding the biological significance of the role of long ncRNAs in infection biology.

Keywords: long non-coding RNA, infection, pathogen, immune, host-pathogen interactions

\section{BACKGROUND}

Recent large-scale, high-resolution transcriptome analyses of several vertebrate genomes, including that of humans, has confirmed the transcriptional potential of large tracts of the vertebrate genome and further opened up new vistas in the study of transcriptomics, RNAs, and their subclasses (Lee and Schatz, 2012). One recent aspect of transcriptomics which has notched up immense interest amongst researchers is the class of RNA's which do not have the potential to code for proteins. This class of RNA's have been dubbed as the non-coding RNAs (ncRNAs) and a subset of this class are the long ncRNAs (long ncRNAs). This recently characterized class (Hung and Chang, 2010) is composed of longer (>200 nt) transcripts and encompasses a number of previously annotated classes of non-coding transcripts such as antisense, lincRNA, processed pseudogenes, and others (Kapranov and St Laurent, 2012). The general features which have been established for long ncRNAs includes their being encoded by RNA Polymerase II and that are capped, polyadenylated like protein-coding transcripts. However, recent reports, including one from our group, suggests a distinct epigenetic regulation of long ncRNAs in comparison to protein-coding genes (Sati et al., 2012). The present estimates for the number of long ncRNAs has crossed 18,000 loci, and extends to cover a number of functionally and mechanistically distinct sub-types.

Despite the scientifically proven functional relevance of long ncRNAs, only a few of them have been well characterized and studied for their roles in the regulation of gene expression. Further, only a handful of articles published to date study the mechanism through which these ncRNAs exert their critical role in several biological scenarios such as genomic imprinting, epigenetic modification, and post-transcriptional regulation of genes. However, given their relevance within the biological machinery as well as hard to ignore significance numerous conceptual mechanistic approaches have been presented to explain their functionality. These range from their possible interaction with other biomolecules within cells to processing into smaller regulatory RNAs (Jalali et al., 2012) and several others (Wang and Chang, 2011).

In comparison to long ncRNAs, most of the RNA subclasses within the ncRNA family have been extensively studied with respect to their roles in viral pathogenesis, antiviral functions, host-pathogen cross talk, immuno-regulatory functions, etc. (Scaria et al., 2006; Rederstorff and Hüttenhofer, 2010). Only a few reports cover the role of long ncRNAs in infection biology inspite of their implicated role in several disease processes such as cancer, psoriasis, Crohn's disease, and many others. Given the significant role of these RNAs in biomolecular regulatory interactions within cells and their prospects as drugs targets in numerous diseased conditions, it becomes highly pertinent and imperative to perform an exhaustive evaluation of their functionalities so as to provide a clear picture of their role in the cellular regulatory machinery and disease processes (Yan and Wang, 2012).

Although the study of the role of long ncRNAs in infections has come a long way from the preliminary studies on viral transcript interactions with cellular components (Keene, 1985), the growth rate in the understanding of long non-coding RNA functionality in infections has not been proportionate to that for other RNAs. 
The present commentary chronicles the role of long ncRNAs in infection biology, with special focus on integrating evidence of their functional role within the same at multiple levels as well as suggesting the potential way forward in examining and unraveling the distinct role of long ncRNAs in several aspects of infection, immunity and host-pathogen cross talk.

\section{LONG NON-CODING RNA CHARACTERIZATION, BIOGENESIS, PROCESSING, AND FUNCTION}

The initial set of long ncRNAs were identified and characterized from the EST libraries present for humans and other model organisms such as Mouse, owing to the persistent and largescale initiatives such as the H-Invitational Database (Yamasaki et al., 2010) and the FANTOM consortium (Ravasi et al., 2010). Following this was the annotation of the transcriptional potential of the genome through tiling microarrays (Kapranov et al., 2003), that provided deep insights into the hidden transcriptional potential of the genome. Furthermore, the recent availability of high-throughput sequencing techniques and transcriptome analysis upto single nucleotide resolution, have added to the number of annotated non-coding RNA loci. The present Gencode annotation includes over 18,000 long ncRNAs. It is generally perceived that most of the long ncRNAs are transcribed by RNA Pol II, with few exceptions. While lncRNAs are known to be essential components of epigenetic gene expression modulation, there exists a limited amount of data on the epigenetic regulation of lncRNA. However, a recent study has pointed toward the similarity of epigenetic regulation of lncRNAs to that of mRNA except for its dissimilarity with regard to DNA methylation (Sati et al., 2012). Further evidence from our group highlights the origin of several novel and previously functionally characterized small RNA from lncRNAs and in most cases from the ends of lncRNAs (Jalali et al., 2012). These results point out to the antecedent of functional small RNA clusters from lncRNA and hence, the potential regulatory role of long non-coding RNA in biological realms. The functions of long ncRNAs are distinct and modulated through biomolecular interactions with other cellular moieties that have been reviewed in detail by Lipovich et al. (2010) and participate in a wide spectrum of regulatory interactions ranging from antisense regulation to epigenetic modification.

\section{LONG NON-CODING RNA EXPRESSION IN RESPONSE TO INFECTION}

Recent reports have increasingly suggested the potential functional consequences of long ncRNAs in infection biology owing to the dysregulation of these ncRNAs during infection processes mostly in response to viral pathogens. One study by Saha et al. (2006) highlighted the role of NEAT1, previously annotated as the Virus Inducible non-coding RNA (VINC1), in the mouse brain following infection with the Japanese Encephalitis virus. Followup studies on the same long non-coding RNA have emphasized the role of NEAT1 in paraspeckle formation and has been further speculated to be a key component in the host responses to viral infections such as the Japanese Encephalitis. Similarly another long non-coding RNA, namely, Psoriasis susceptibility-related RNA Gene Induced by Stress (PRINS) has been shown to be upregulated following infection with the Herpes Simplex Virus (Sonkoly et al., 2005) and treatment with bacterial cell wall extracts (Bari et al., 2011) apart from other stress factors such as ultraviolet radiation, etc. Unbiased genome-wide associations have also implicated PRINS as a conspicuous factor in psoriasis (Sonkoly et al., 2005). Further, a recent report by Peng et al. (2010) has indicated distinct signatures of long non-coding RNA expression in a SARS infection model and significant dysregulation in response to infection. Similar responses were also obtained post-treatment with interferons, indicating the possibility of a common pathway for infection response and long non-coding RNA regulation mediated through the interferon gamma involved immunological pathways. The role of long non-coding RNA in infections has not been restricted solely to animals but also extended to those in plants and model systems like Dictyostelium. In the model organism Dictyostelium discoideum, a long non-coding RNA DutA (Development-specific but UnTranslatable RNA A) has been determined to be dysregulated post-infection (Farbrother et al., 2006), while in plant systems, the PINCI1 family has been identified to be upregulated following P. infestans infection in potato (Avrova et al., 2007).

\section{LONG NON-CODING RNAs AND IMMUNE CELLS}

One of the major determinants of infection biology in higher organisms is an intact immune system, the absence of which marks an immunocompromised condition. Several studies have been conducted to date with the aim of unraveling the role of noncoding RNA regulatory networks in the overall infection process, particularly those which are related to microRNAs. Recent reports have seen widespread attention on long non-coding RNA based regulation in immune cells and immunological responses. Pang et al. (2009) utilized sequencing based transcriptome analysis to characterize the transcriptome and determine the number of long ncRNAs that are expressed in CD8+ T-cells. Additional in-depth analyses also suggested that many of these long non-coding RNA loci share their genomic loci with protein-coding genes, with some of these overlapping small RNA sites, thereby indicating the possible mechanism through which the processing of a subset of long ncRNAs takes place. An example of the role of long noncoding RNA in immunological function is that of TMEVPG1 (Theiler's murine encephalomyelitis virus persistence candidate gene 1) which has been shown to be expressed post-infection in the CD4 and CD8+ T-cells and downregulated following immune cell activation (Vigneau et al., 2003) possibly through the regulation of IFN gamma.

\section{EVIDENCE FROM GENOME-WIDE ASSOCIATIONS}

The development of unbiased genome-wide assays for genetic markers associated with several traits has contributed immensely to the understanding of the mechanizations underlying the role of long ncRNAs in disease processes. Several studies have utilized genome-wide associations to enunciate the relationship of long non-coding RNA loci with progression to Hepatocellular Carcinoma following infection with Hepatitis. One of these studies has identified the genomic SNP rs7763881, mapping to HULC loci, as causative of conferring decreased susceptibility to Hepatocellular carcinomas in HBV (Liu et al., 2012) while another has indicated four SNPS in 8p12, which map to a long non-coding RNA loci, as conferring increased susceptibility to infection in certain patients 
(Chan et al., 2011). Two similar studies for HIV have indicated an endogenous retroviral locus that codes for a long non-coding RNA as a genetic loci for susceptibility to infection with HIV (Dalmasso et al., 2008; Limou et al., 2009).

\section{PATHOGEN ENCODED LONG NON-CODING RNAs}

Pathogen genomes have also been confirmed to encode for long ncRNAs with several viruses encoding for long ncRNAs. This includes the EBER1 and EBER2 RNAs encoded by the EpsteinBarr Virus that have been determined to be associated with the particular viruses latency and malignancy (Nanbo et al., 2005; Iwakiri et al., 2009; Iwakiri and Takada, 2010); beta 2.7 RNA encoded by the Cytomegalovirus, which prevents the host cell from undergoing apoptosis following infection and ensuring cell survivability conducive to the virus (Reeves et al., 2007; Zhao et al., 2010); HSUR1 and HSUR2 encoded by the Herpesvirus genome, that mimics the target for the microRNA mir-27 and hence, modulates the T-cell gene expression (Cook et al., 2005; Buck et al., 2010; Cazalla et al., 2010) and lastly, VAI and VAII coded for by the human adenovirus and implicated as key determinants in the suppression of host cell-derived RNAi response following viral replication (Andersson et al., 2005; McKenna et al., 2006; Sano et al., 2006; Xu et al., 2007). In a peculiar case, as exemplified in the case of $\mathrm{HBZ}$ encoded by HTLV-1, increasing evidence suggests apart from coding for a protein, the mRNA could have non-coding functions (Chaudhary and Ratner, 2011; Nakano and Watanabe, 2012) with distinct nuclear retention and possible effect in persistence and immune evasion (Rende et al., 2011). Apart from viruses, long ncRNAs have been also identified in the pathogen, Plasmodium falciparum wherein a genome-wide transcriptome annotation by Broadbent et al. (2011) showed the presence of 22 Telomere-associated ncRNAs. These lncRNAs were found to be localized to the perinuclear compartment of the pathogen (Sierra-Miranda et al., 2012) and contributing to its biology by possible interaction with nuclear proteins.

\section{LONG NON-CODING RNAs IN HOST-PATHOGEN INTERACTION}

At present the mechanistic understanding of the role of lncRNAs in or following infection and host responses to infection may at best be described as being sketchy and limited to a few studies and even more so, primarily to four models only. Each of these reports presents an interesting new opportunity to understand and evaluate the function and interaction of long non-coding RNA following infection. Mechanistically, dysregulation of long ncRNAs could modulate downstream regulation of genes at several functional levels ranging from epigenetic changes influencing chromatin organization to post-transcriptional regulation at transcript levels as well as through direct interaction with other biomolecules such as proteins and RNAs (Wang and Chang, 2011; Moran et al., 2012). These interactions could modulate (a) host responses to an invading pathogen not excluding immunological mechanisms (b) regulation of pathogen growth and replication (c) regulation of cellular death/apoptosis or survival (d) general stress responses. While considerable contention exists over the exact mechanism through which viral

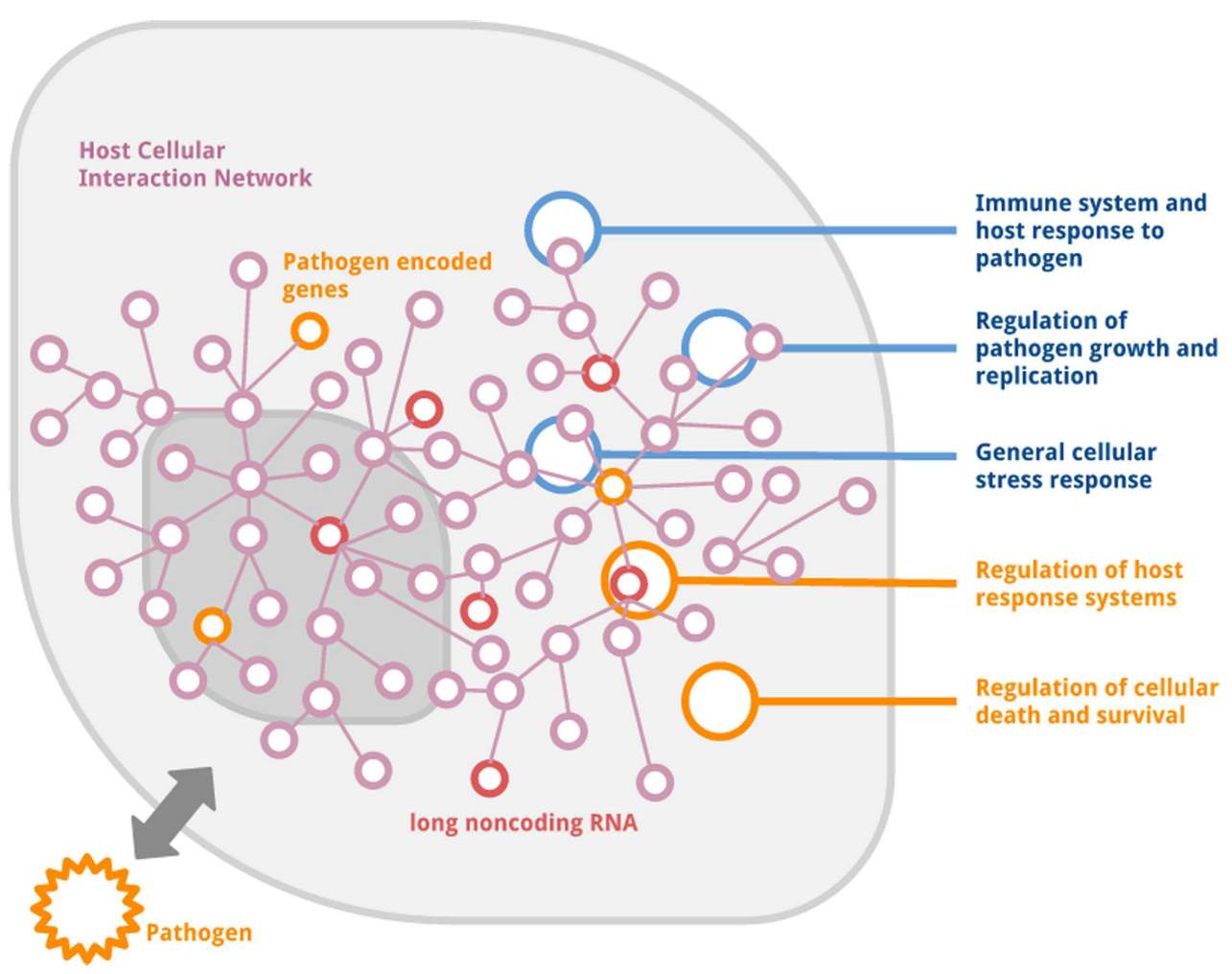

FIGURE 1 | Schematic of the role of long non-coding RNAs in host-pathogen interaction networks. 
lncRNAs act, it has been suggested that the viral long ncRNAs potentially exploit the interaction networks within hosts, thereby affecting their response to infections in an attempt to evade the immunological onslaught. They do this through a variety of mechanisms, including the inhibition of RNAi response or target mimicry (Franko-Zorilla et al., 2007). The schematic overview of the reported mechanisms through which long ncRNAs mediate in host-pathogen interactions has been summarized in Figure 1.

\section{GENOME-SCALE BIOLOGY AND OPPORTUNITIES IN UNDERSTANDING ROLE OF LONG NON-CODING RNAs IN INFECTION AND HOST RESPONSE}

Recent technological advancements in the analytical techniques for transcriptomic evaluation has opened up new vistas in the possibilities through which the function, interaction, and regulatory mechanisms of lncRNAs may be understood at the genomic level. Further developments in computational methodologies have enabled researchers to functionally characterize lncRNA through the interpretation and analysis of genome-scale transcriptome correlations. These improvements have been aided in part from the generation and availability of large central repositories containing data pertinent to large-scale experiments which may be utilized to integrate datasets with the aim of understanding long noncoding RNA biology. Recent reports on the integration of datasets on disparate transcriptomes have provided an immense impetus to the understanding of functional consequence of long ncRNAs in cancers (Brunner et al., 2012). These interpretations could be further extrapolated to evaluate specific signatures and hence identify the long ncRNAs that are involved in infections and host immune-responses consequent to infection. Finally, the availability of similar technologies to understand protein-RNA interactions (Yeo et al., 2009; Hafner et al., 2010; Wen et al., 2011; Ascano et al., 2012; Scheibe et al., 2012) and epigenetic modifications during infection processes also opens up vast new opportunities in the understanding of the biology and regulation of long ncRNAs during infection.

The conclusions derived from the studies conducted to date as well as further evaluations would provide novel approaches for implementation in drug development and discovery through the design of targeted strategies that would specifically aim biomolecular interactions mediated by long ncRNAs.

However, the specific targeting of RNAs using small molecules would necessitate an accurate estimation of RNA structure that would in part be aided by recent techniques such as SHAPE (McGinnis et al., 2012) and PARE (Yu et al., 2007) that offer attractive possibilities in the understanding of RNA structure for

\section{REFERENCES}

Amit, I., Garber, M., Chevrier, N., Leite, A. P., Donner, Y., Eisenhaure, T., et al. (2009). Unbiased reconstruction of a mammalian transcriptional network mediating the differential response to pathogens. Science 326, 257-263.

Andersson, M. G., Haasnoot, P. C., Xu, N., Berenjian, S., Berkhout, B., and Akusjärvi, G. (2005). Suppression

larger lengths of transcripts. Recent studies have highlighted the distribution of specific regulatory motifs like $G$ quadruplexes in RNA, which may serve as specific targets for RNAs (Biffi et al., 2012; Weng et al., 2012).

\section{FUTURE PERSPECTIVE}

Based on the literary evidence to date, it is apparent that lncRNAs play a significant role in the overall infection and immunological process. Several viral lncRNAs such as EBER1, EBER2, VAI, VAII, etc., have been positively ascertained to play a significant role in increasing the pathogen survivability within the host. While on the other hand, numerous RNAs present in humans have also been identified to play a critical role in host immunological responses (such as the TMEVPG1, HUCL, etc.) influencing the overall outcome of infections depending upon their ability to either increase or decrease patient susceptibility to infections (Vigneau et al., 2003; Dalmasso et al., 2008; Limou et al., 2009; Chan et al., 2011; Liu et al., 2012). In view of the consequential role of lncRNA in infection biology, it is imperative to completely elucidate their complete function and regulatory role post-infections and analyze the bottlenecks within. This has in part been aided by the recent leaps in transcriptome technology and data generation, however, there is still an immense scope within the field for the same. Recent studies by Amit et al. (2009) and Shapira et al. (2009) provide interesting insights into the role of RNA machinery and factors involved in host-pathogen interaction following infection. While the former puts forward an unbiased approach for elucidating the mechanisms through which regulatory networks control transcriptional response post-infections, the latter explores the host-pathogen factors and pathways involved through which the H1N1 virus exerts its effect on host systems subsequent to infection. Once understood, these lncRNAs may serve as drug targets that may be utilized for further drug development as well as to study the host-pathogen interplay following infection, thereby increasing the possibility of improving patient prognosis post-infection.

\section{ACKNOWLEDGMENTS}

The authors acknowledge scientifically stimulating discussions with members of Vinod Scaria Lab. Authors also thank Drs. Chetana Sachidanandan and Sridhar Sivasubbu for discussions and reviewing the manuscript and Ms. Deeksha Bhartiya for compiling the long non-coding RNAs associated with GWAS markers. Ayesha Pasha acknowledges a fellowship from the Open Source Drug Discovery (OSDD) Programme. This work was funded by the CSIR Open Source Drug Discovery (OSDD) Programme through grant number HCP001.

I., et al. (2007). A novel nonprotein-coding infection-specific gene family is clustered throughout the genome of Phytophthora infestans. Microbiology 153(Pt 3), 747-759.

Bari, L., Bacsa, S., Sonkoly, E., BataCsörgo, Z., Kemény, L., Dobozy, A., et al. (2011). Comparison of stressinduced PRINS gene expression in normal human keratinocytes and
HaCaT cells. Arch. Dermatol. Res. 303, 745-752.

Biffi, G., Tannahill, D., and Balasubramanian, S. (2012). An intramolecular G-quadruplex structure is required for binding of telomeric repeat-containing RNA to the telomeric protein TRF2. J. Am. Chem. Soc. 134, 11974-11976.

Broadbent, K. M., Park, D., Wolf, A. R., Van Tyne, D., Sims, J. S., Ribacke, U., 
et al. (2011). A global transcriptional analysis of Plasmodium falciparum malaria reveals a novel family of telomere-associated lncRNAs. Genome Biol. 12, R56.

Brunner, A. L., Beck, A. H., Edris, B., Sweeney, R. T., Zhu, S. X., Li, R., et al. (2012). Transcriptional profiling of lncRNAs and novel transcribed regions across a diverse panel of archived human cancers. Genome Biol. 13, R75.

Buck, A. H., Perot, J., Chisholm, M. A., Kumar, D. S., Tuddenham, L., Cognat, V., et al. (2010). Posttranscriptional regulation of $\mathrm{miR}$ 27 in murine cytomegalovirus infection. RNA 16, 307-315.

Cazalla, D., Yario, T., and Steitz, J. A. (2010). Down-regulation of a host microRNA by a Herpesvirus saimiri noncoding RNA. Science 328, 1563-1566.

Chan, K. Y., Wong, C. M., Kwan, J. S., Lee, J. M., Cheung, K. W., Yuen, M. F., et al. (2011). Genome-wide association study of hepatocellular carcinoma in Southern Chinese patients with chronic hepatitis B virus infection. PLoS ONE 6:e28798. doi:10.1371/journal.pone.0028798

Chaudhary, G., and Ratner, L. (2011). The HTLV-1 hbz antisense gene indirectly promotes tax expression via down-regulation of $\mathrm{p} 30$ (II) mRNA. Virology 410, 307-315.

Cook, H. L., Lytle, J. R., Mischo, H. E., Li, M. J., Rossi, J. J., Silva, D. P., et al. (2005). Small nuclear RNAs encoded by Herpesvirus saimiri upregulate the expression of genes linked to $\mathrm{T}$ cell activation in virally transformed T cells. Curr. Biol. 15, 974-979.

Dalmasso, C., Carpentier, W., Meyer, L., Rouzioux, C., Goujard, C., Chaix, M. L., et al. (2008). Distinct genetic loci control plasma HIV-RNA and cellular HIV-DNA levels in HIV-1 infection: the ANRS Genome Wide Association 01 study. PLoS ONE 3:e3907. doi:10.1371/journal.pone.0003907

Farbrother, P., Wagner, C., Na, J., Tunggal, B., Morio, T., Urushihara, H., et al. (2006). Dictyostelium transcriptional host cell response upon infection with Legionella. Cell. Microbiol. 8, 438-456.

Franko-Zorilla, J. M., Valli, A., Todesco, M., Mateos, I., Puga, M. I., Rubio-Somoza, I., et al. (2007). Target mimicry provides a new mechanism for the regulation of microRNA activity. Nat. Genet. 39, 1033-1037.

Hafner, M., Landthaler, M., Burger, L., Khorshid, M., Hausser, J., Berninger, P., et al. (2010). PAR-CliP - a method to identify transcriptome-wide the binding sites of RNA binding proteins. J. Vis. Exp. 41, pii 2034. doi: $10.3791 / 2034$

Hung, T., and Chang, H. Y. (2010). Long noncoding RNA in genome regulation: prospects and mechanisms. RNA Biol. 7, 582-585.

Iwakiri, D., and Takada, K. (2010). Role of EBERs in the pathogenesis of EBV infection. Adv. Cancer Res. 107, 119-136.

Iwakiri, D., Zhou, L., Samanta, M., Matsumoto, M., Ebihara, T., Seya, T., et al. (2009). Epstein-Barr virus (EBV)-encoded small RNA is released from EBV-infected cells and activates signaling from Tolllike receptor 3. J. Exp. Med. 206, 2091-2099.

Jalali, S., Gunanathan, G., and Scaria, V. (2012). Integrative transcriptome analysis suggest processing of a subset of long non-coding RNAs to small RNAs. Biol. Direct 7, 25.

Kapranov, P., Sementchenko, V. I., and Gingeras, T. R. (2003). Beyond expression profiling: next generation uses of high density oligonucleotide arrays. Brief. Funct. Genomic. Proteomic. 2, 47-56.

Kapranov, P., and St Laurent, G. (2012). Dark matter RNA: existence, function, and controversy. Front. Genet. 3:60. doi:10.3389/fgene.2012.00060

Keene, J. D. (1985). Interactions between small viral RNAs of vesicular stomatitis virus and components of cellular gene expression. Microbiol. Sci. 2, 152-156.

Lee, H., and Schatz, M. C. (2012). Genomic dark matter: the reliability of short read mapping illustrated by the genome mappability score. Bioinformatics 28, 2097-2105.

Limou, S., Le Clerc, S., Coulonges, C., Carpentier, W., Dina, C., Delaneau, O., et al. (2009). Genomewide association study of an AIDSnonprogression cohort emphasizes the role played by HLA genes (ANRS genomewide association study 02 ). J. Infect. Dis. 199, 419-426.

Lipovich, L., Johnson, R., and Lin, C. Y. (2010). MacroRNA underdogs in a microRNA world: evolutionary, regulatory, and biomedical significance of mammalian long non-proteincoding RNA. Biochim. Biophys. Acta 1799, 597-615.

Liu, Y., Pan, S., Liu, L., Zhai, X., Liu, J., Wen, J., et al. (2012). A genetic variant in long non-coding RNA HULC contributes to risk of HBV-related hepatocellular carcinoma in a Chinese population. PLoS ONE 7:e35145. doi:10.1371/ journal.pone.0035145
McGinnis, J. L., Dunkle, J. A., Cate, J. H. and Weeks, K. M. (2012). The mechanisms of RNA SHAPE chemistry. $J$. Am. Chem. Soc. 134, 6617-6624.

McKenna, S. A., Kim, I., Liu, C. W., and Puglisi, J. D. (2006). Uncoupling of RNA binding and PKR kinase activation by viral inhibitor RNAs. J. Mol. Biol. 358, 1270-1285.

Moran, V. A., Perera, R. J., and Khalil, A. M. (2012). Emerging functional and mechanistic paradigms of mammalian long non-coding RNAs. Nucleic Acids Res. 40, 6391-6400.

Nakano, K., and Watanabe, T. (2012). HTLV-1 Rex: the courier of viral messages making use of the host vehicles. Front. Microbiol. 3:330. doi:10.3389/fmicb.2012.00330

Nanbo, A., Yoshiyama, H., and Takada, K. (2005). Epstein-Barr virusencoded poly(A)- RNA confers resistance to apoptosis mediated through Fas by blocking the PKR pathway in human epithelial intestine 407 cells. J. Virol. 79, 12280-12285.

Pang, K. C., Dinger, M. E., Mercer, T. R., Malquori, L., Grimmond, S. M., Chen, W., et al. (2009). Genomewide identification of long noncoding RNAs in CD8+ T cells. $J$. Immunol. 182, 7738-7748.

Peng, X., Gralinski, L., Armour, C. D. Ferris, M. T., Thomas, M. J., Proll, S., et al. (2010). Unique signatures of long noncoding RNA expression in response to virus infection and altered innate immune signaling. MBio 1, e00206-e00210.

Ravasi, T., Suzuki, H., Cannistraci, C.V. Katayama, S., Bajic, V. B., Tan, K., et al. (2010). An atlas of combinatorial transcriptional regulation in mouse and man. Cell 140, 744-752.

Rederstorff, M., and Hüttenhofer, A. (2010). Small non-coding RNAs in disease development and hostpathogen interactions. Curr. Opin. Mol. Ther. 12, 684-694.

Reeves, M. B., Davies, A. A., McSharry, B. P., Wilkinson, G. W., and Sinclair, J. H. (2007). Complex I binding by a virally encoded RNA regulates mitochondria-induced cell death. Science 316, 1345-1348.

Rende, F., Cavallari, I., Corradin, A. Silic-Benussi, M., Toulza, F., Toffolo, G. M., et al. (2011). Kinetics and intracellular compartmentalization of HTLV-1 gene expression: nuclear retention of HBZ mRNAs. Blood 117, 4855-4859.

Saha, S., Murthy, S., and Rangarajan, P. N. (2006). Identification and characterization of a virus-inducible non-coding RNA in mouse brain. $J$. Gen. Virol. 87(Pt 7), 1991-1995.
Sano, M., Kato, Y., and Taira, K. (2006). Sequence-specific interference by small RNAs derived from adenovirus VAI RNA. FEBS Lett. 580, 1553-1564.

Sati, S., Ghosh, S., Jain, V., Scaria, V., and Sengupta, S. (2012). Genomewide analysis reveals distinct patterns of epigenetic features in long non-coding RNA loci. Nucleic Acids Res. 40, 10018-10031.

Scaria, V., Hariharan, M., Maiti, S., Pillai, B., and Brahmachari, S. K. (2006) Host-virus interaction: a new role for microRNAs. Retrovirology 3:68. doi:10.1186/1742-4690-3-68

Scheibe, M., Butter, F., Hafner, M., Tuschl, T., and Mann, M. (2012). Quantitative mass spectrometry and PAR-CLIP to identify RNA-protein interactions. Nucleic Acids Res. 40, 1-6.

Shapira, S. D., Gat-Viks, I., Shum, B.O.V., Dricot, A., de Grace, M. M., Wu, L. (2009). A physical and regulatory map of host-influenza interactions reveals pathways in $\mathrm{H} 1 \mathrm{~N} 1$ infection. Cell 139, 1255-1267.

Sierra-Miranda, M., Delgadillo, D. M., Mancio-Silva, L., Vargas, M., Villegas-Sepulveda, N., MartínezCalvillo, S., et al. (2012). Two long non-coding RNAs generated from subtelomeric regions accumulate in a novel perinuclear compartment in Plasmodium falciparum. Mol. Biochem. Parasitol. 185, 36-47.

Sonkoly, E., Bata-Csorgo, Z., Pivarcsi, A., Polyanka, H., Kenderessy-Szabo, A., Molnar, G., et al. (2005). Identification and characterization of a novel, psoriasis susceptibility-related noncoding RNA gene, PRINS. J. Biol. Chem. 280, 24159-24167.

Vigneau, S., Rohrlich, P. S., Brahic, M., and Bureau, J. F. (2003). Tmevpg1, a candidate gene for the control of Theiler's virus persistence, could be implicated in the regulation of gamma interferon. J. Virol. 77, 5632-5638.

Wang, K. C., and Chang, H. Y. (2011). Molecular mechanisms of long noncoding RNAs. Mol. Cell 43, 904-914.

Wen, J., Parker, B. J., Jacobsen, A., and Krogh, A. (2011). MicroRNA transfection and AGO-bound CLIP-seq data sets reveal distinct determinants of miRNA action. RNA 17, 820-834.

Weng, H. Y., Huang, H. L., Zhao, P. P., Zhou, H., and Qu, L. H. (2012). Translational repression of cyclin D3 by a stable G-quadruplex in its $5^{\prime}$ UTR: implications for cell cycle regulation. RNA Biol. 9, 1099-1109. 
Xu, N., Segerman, B., Zhou, X., and Akusjärvi, G. (2007). Adenovirus virus-associated RNAIIderived small RNAs are efficiently incorporated into the RNA-induced silencing complex and associate with polyribosomes. J. Virol. 81, 10540-10549.

Yamasaki, C., Murakami, K., and Takeda, J. (2010). H-InvDB in 2009: extended database and data mining resources for human genes and transcripts. Nucleic Acids Res. 38, D626-D632.

Yan, B., and Wang, Z. (2012). Long noncoding RNA: its physiological and pathological roles. DNA Cell Biol. 31(Suppl. 1), S34-S41.

Yeo, G. W., Coufal, N. G., Liang, T. Y., Peng, G. E., Fu, X. D., and Gage, F. H. (2009). An RNA code for the FOX2 splicing regulator revealed by mapping RNA-protein interactions in stem cells. Nat. Struct. Mol. Biol. 16, 130-137.

Yu, E. Z., Burba, A. E., and Gerstein, M. (2007). PARE: a tool for comparing protein abundance and mRNA expression data. BMC Bioinformatics 8:309. doi:10.1186/1471-2105-8-309

Zhao, J., Sinclair, J., Houghton, J., Bolton, E., Bradley, A., and Lever,
A. (2010). Cytomegalovirus beta2.7 RNA transcript protects endothelial cells against apoptosis during ischemia/reperfusion injury. J. Heart Lung Transplant. 29, 342-345.

Conflict of Interest Statement: The authors declare that the research was conducted in the absence of any commercial or financial relationships that could be construed as a potential conflict of interest.

Received: 14 October 2012; accepted: 12 December 2012; published online: 09 January 2013.
Citation: Scaria $V$ and Pasha A (2013) Long non-coding RNAs in infection biology. Front. Gene. 3:308. doi: 10.3389/fgene.2012.00308

This article was submitted to Frontiers in Non-Coding RNA, a specialty of Frontiers in Genetics.

Copyright $\odot 2013$ Scaria and Pasha. This is an open-access article distributed under the terms of the Creative Commons Attribution License, which permits use, distribution and reproduction in other forums, provided the original authors and source are credited and subject to any copyright notices concerning any third-party graphics etc. 